\title{
Low-Energy Muons as a Tool for a Depth-Resolved Analysis of the $\mathrm{SiO} / 4 \mathrm{H}-\mathrm{SiC}$ Interface
}

\section{Conference Paper}

\section{Author(s):}

Woerle, Judith (D); Prokscha, Thomas; Grossner, Ulrike (D)

Publication date:

2020-07

Permanent link:

https://doi.org/10.3929/ethz-b-000430948

Rights / license:

In Copyright - Non-Commercial Use Permitted

Originally published in:

Materials Science Forum 1004, https://doi.org/10.4028/www.scientific.net/msf.1004.581 


\title{
Low-Energy Muons as a Tool for a Depth-Resolved Analysis of the $\mathrm{SiO}_{2} / 4 \mathrm{H}-\mathrm{SiC}$ Interface
}

\author{
Judith Woerle ${ }^{1, a^{*}}$, Thomas Prokscha ${ }^{2, b}$ and Ulrike Grossner ${ }^{1, c}$ \\ ${ }^{1}$ Advanced Power Semiconductor Laboratory, ETH Zurich, Physikstrasse 3, 8092 Zurich, \\ Switzerland \\ 2Laboratory for Muon Spin Spectroscopy, Paul Scherrer Institute, 5232 Villigen PSI, Switzerland \\ awoerle@aps.ee.ethz.ch, bthomas.prokscha@psi.ch, culrike.grossner@ethz.ch
}

Keywords: muon spin rotation spectroscopy, depth-profile, oxidation, interface defects

\begin{abstract}
In this work, the potential of muon spin rotation $(\mu \mathrm{SR})$ with low-energy muons (LE- $\mu$ ) for the investigation of oxidation-induced defects at the $\mathrm{SiO}_{2} / 4 \mathrm{H}-\mathrm{SiC}$ interface is explored. By using implantation energies for the muons in the $\mathrm{keV}$ range and comparing the fractions of muonium in different regions, the depth distribution of defects in the first $200 \mathrm{~nm}$ of the target material can be resolved. Defect profiles of interfaces with either deposited or thermally grown $\mathrm{SiO}_{2}$ layers on $4 \mathrm{H}-$ $\mathrm{SiC}$ are compared. The results show an increased number of defects in the case of a thermal oxide, both on the oxide and on the $\mathrm{SiC}$ side of the interface, with a spatial extension of a few tens of nm.
\end{abstract}

\section{Introduction}

Thermal oxidation of silicon carbide $(\mathrm{SiC})$ is known to generate a large density of interface defects $\left(D_{\text {it }}\right)$, leading to threshold-voltage instabilities and low channel mobilities of SiC MOSFETs $[1,2]$. While the energy position of electrically active traps has been intensely studied, their spatial position with respect to the $\mathrm{SiO}_{2} / \mathrm{SiC}$ interface is less understood. Based on the electrical analysis of MOS capacitors using capacitance or conductance methods as well as photoemission (PE) or electron energy loss spectroscopy (EELS), these interface traps are assumed to reside in a substoichiometric, defect-rich transition region on the oxide side of the interface. There is, however, a large spread of experimental data on the extension of this sub-stoichiometric region and reports range from several Å to tens of nm [3-6].

Muon spin rotation is an experimental technique that is based on the implantation of spinpolarized muons and is commonly employed to study magnetic fields inside the target material at the atomic scale $[7,8]$. After a mean lifetime of $2.2 \mu \mathrm{s}$, the muons decay into a positron $\mathrm{e}^{+}$and two neutrinos. By measuring the direction of the emitted positrons in a time-resolved fashion with detectors placed around the sample, conclusions on the interaction of the muons with the sample's local environment can be drawn. Although hydrogen or hydrogen-related impurities are by far the most studied type of semiconductor defects in $\mu \mathrm{SR}$, it was recently shown that this technique also allows the investigation of other defect types such as surface defects in quaternary solar cell materials [9].

In this work, we present first results where LE- $\mu$ SR is used to study the depth distribution of defects at the $\mathrm{SiO}_{2} / 4 \mathrm{H}-\mathrm{SiC}$ interface. The interface formation during two oxidation processes thermal oxidation and $\mathrm{SiO}_{2}$ deposition using a plasma-enhanced chemical vapor deposition (PECVD) tool are compared.

\section{Working principle of $\mu \mathrm{SR}$}

Positive muons are a decay product of pions, which are generated when high-energy protons $(>500 \mathrm{MeV})$ collide with the nuclei of a production target (e.g. $\mathrm{C}$ or Be). They have a spin of 1/2, a 
mass of $207 m_{\mathrm{e}}$ (or $1 / 9 m_{\mathrm{p}}$ ) and due to the parity violating nature of the pion decay, are spinpolarized.

A sketch of a typical $\mu$ SR setup is shown in Fig. 1. When the $\mu^{+}$are implanted into the sample, they quickly lose energy, adopt interstitial sites of the crystal lattice and thermalize without any significant loss of polarization. In the presence of a magnetic field, in our case applied perpendicular to the initial muon polarization, the spins of the implanted muons precess at the Larmor frequency $\omega_{\mu}=\gamma_{\mu} \cdot \mathrm{B}\left(\gamma_{\mu} \cdot \mathrm{B}=2 \pi \cdot 136 \mathrm{MHz} / \mathrm{T}\right)$. The field at the muon stopping site is then the vector sum of the applied field and any local field present in the sample. Once the muons decay with a mean lifetime of $\sim 2.2 \mu \mathrm{s}$, the $\mathrm{e}^{+}$are preferentially emitted along the direction of the muon spin at the time of the decay and detected by the scintillator plates.

The implantation of $\mu^{+}$into insulators or semiconductors leads to the formation of a hydrogenlike bound state called Muonium $\left[\mathrm{Mu}^{0}=\left(\mu^{+} \mathrm{e}^{-}\right)\right] \cdot \mathrm{Mu}^{0}$ has a $\sim 103$ times larger muon precession frequency due to the hyperfine coupling between $\mu^{+}$and the electron spin, which allows to distinguish between diamagnetic (unbound $\mu^{+}$) and paramagnetic $\left(\mathrm{Mu}^{0}\right)$ contributions. The $\mu^{+}$and $\mathrm{Mu}^{0}$ decay symmetries $A_{\mathrm{D}}$ and $A_{\mathrm{Mu}}$ are determined by the amplitude of the corresponding precession signals and are proportional to the fraction of muons in a particular state.

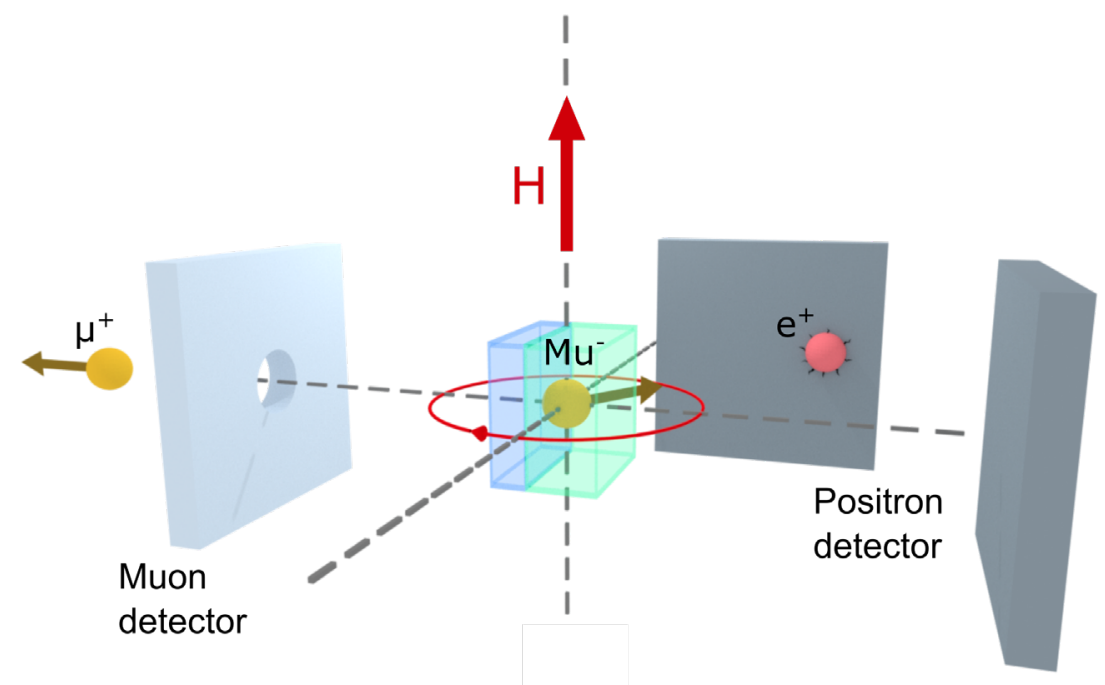

Fig. 1. Schematic of a transverse field (TF) $\mu \mathrm{SR}$ setup used for studying the $\mathrm{SiO}_{2} / 4 \mathrm{H}-\mathrm{SiC}$ interface. In this sketch, the muons are entering from the left. The brown arrows indicate the spin of the muon, which is opposite to its flight direction.

As it was previously shown, the fraction of implanted muons forming $\mathrm{Mu}^{0}$ changes in materials with large structural disorder [10-13]. Conventional $\mu$ SR techniques, however, use implantation energies in the $\mathrm{MeV}$ range that do not allow to measure shallow depth profiles with satisfying resolution. In order to extend $\mu \mathrm{SR}$ to device-relevant thin film structures, low-energy muons with kinetic energies in the low $\mathrm{keV}$ range are required. Only recently, a LE- $\mu$ SR study, dedicated to the investigation of point defects in shallow crystal regions of $\mathrm{Si}$ and $\mathrm{SiC}$ was successfully demonstrated [14].

\section{Experimental Details}

For this study, both the epitaxially grown (0001) Si-face (n-type, $30 \mu \mathrm{m}, N_{\mathrm{D}}=2.8 \times 10^{15} \mathrm{~cm}^{-3}$ ) and the highly doped (000-1) C-face (n-type, $N_{\mathrm{D}} \approx 1 \times 10^{19} \mathrm{~cm}^{-3}$ ) of $4 \mathrm{H}-\mathrm{SiC}$ wafers were used. All samples received either a PECVD-deposited $\mathrm{SiO}_{2}$ with an oxide thickness of $t_{\mathrm{ox}}=105 \mathrm{~nm}$ (SiPECVD), or were thermally oxidized in $\mathrm{O}_{2}$ ambient at $1050^{\circ} \mathrm{C}\left(t_{\mathrm{ox}}=85 \mathrm{~nm}\right)$ (C-DRY, Si- 
DRY+PECVD). The Si-face sample with a thermally grown oxide (Si-DRY+PECVD) received an additional PECVD-deposited $\mathrm{SiO}_{2}$ layer to have comparable depths of the interface with respect to the surface. On another C-face sample with a dry oxide, the $\mathrm{SiO}_{2}$ was stripped off again and a $\mathrm{PECVD}_{-\mathrm{SiO}_{2}}\left(t_{\mathrm{ox}}=95 \mathrm{~nm}\right)$ was deposited instead (C-DRY-PECVD). A profilometer was used to determine the final oxide thickness while the oxide densities were extracted with an $\mathrm{x}$-ray diffractometer. An overview of the measured samples is shown in Table 1.

Table 1: Sample overview. All samples used in the study were cut from the same 4H-SiC wafer.

\begin{tabular}{|l|c|c|c|}
\hline Sample Name & Orientation & Oxidation Process & tox (nm) \\
\hline Si-PECVD & $(0001)$ & PECVD & 105 \\
\hline C-DRY & $(000-1)$ & thermal & 90 \\
\hline Si-DRY+PECVD & $(0001)$ & thermal + PECVD & $30+70$ \\
\hline C-DRY-PECVD & $(000-1)$ & $\begin{array}{c}\text { thermal, etch back, } \\
\text { PECVD }\end{array}$ & 95 \\
\hline
\end{tabular}

All muon measurements were performed at the low-energy muon facility (LEM) at the Swiss Muon Source $(\mathrm{S} \mu \mathrm{S})$, Switzerland $[14,15]$. The samples were glued to a Ni-coated holder with silver paste and mounted onto a cryostat placed perpendicular to the muon beam. For the muon implantations, kinetic energies between $3 \mathrm{keV}$ and $20 \mathrm{keV}$ were used to probe depths down to 160 $\mathrm{nm}$ below the sample surface. For all measurements, the FWHM spot size of the muon beam was 12 $\mathrm{mm}$. All measurements where performed at $10 \mathrm{~K}$ and with a magnetic field of $10 \mathrm{mT}$ which was applied parallel to the beam axis. Scintillator detectors placed above, below, left and right of the beam axis were used for the detection of the decay positrons. For each energy point, around $10^{6}$ muon decays were measured.

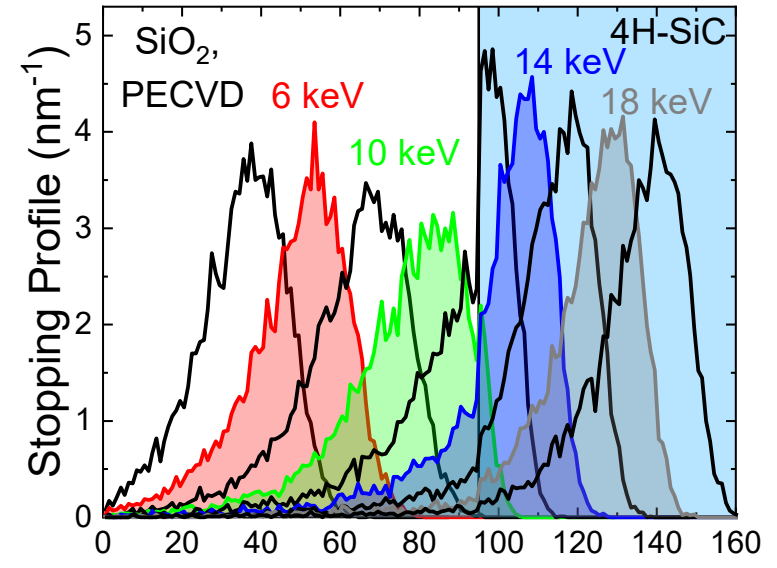

(a)

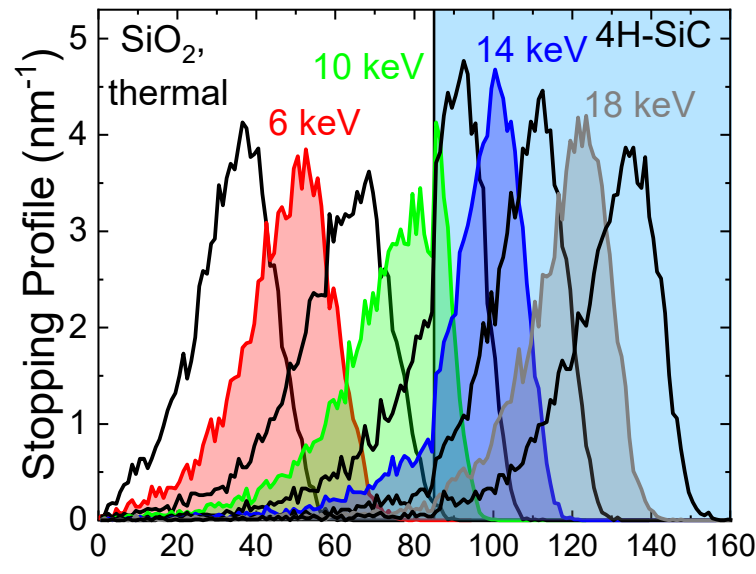

(b)

Depth $(\mathrm{nm})$

Fig. 2. Calculated muon stopping profiles for a low-density deposited $\mathrm{SiO}_{2}$ layer (a) and a thermally grown $\mathrm{SiO}_{2}$ layer (b) on top of $4 \mathrm{H}-\mathrm{SiC}$. For the simulations, Trim.SP was used $[16,17]$. 


\section{Results and Discussion}

Figure 2 shows calculated muon stopping profiles for both oxide types and for the range of muon energies used in this study. As indicated in the graph, implantation energies below $10 \mathrm{keV}$ are mainly probing the oxide, while muons with larger energies also reach into the SiC bulk.

For all samples, the diamagnetic decay asymmetry $A_{D}$ is determined. $A_{\mathrm{D}}$ is proportional to the fractions of muons in a diamagnetic state and decreases with an increasing fraction of $\mathrm{Mu}^{0}$ formation. For the specific setup and measurement parameters, a maximum of $A_{\mathrm{D}} \approx 0.2$ indicates that no $\mathrm{Mu}^{0}$ is formed and all $\mu^{+}$decay as free muons. In the case of the investigated $\mathrm{SiO}_{2} / \mathrm{SiC}$ interface, a decrease of the $\mathrm{Mu}^{0}$ fraction may have different reasons. One possible scenario are oxidation-induced electron traps, causing an electron shortage around the implanted muons and hence a reduced $\mathrm{Mu}^{0}$ formation probability. Another explanation for higher $A_{D}$ values is oxidationinduced lattice strain, which may significantly change the availability of $\mathrm{Mu}^{0}$ lattice sites and with that also the $\mathrm{Mu}^{0}$ fraction in a disordered crystal.

In the case of the deposited $\mathrm{SiO}_{2}$ (Si-PECVD), high asymmetry values are observed at energies below $\sim 12 \mathrm{keV}$. The $\mathrm{Mu}^{0}$ formation is strongly suppressed in the oxide region, pointing to a $\mathrm{SiO}_{2}$ of very low quality. This is not very surprising as no post-deposition annealing for an oxide densification was performed after the PECVD process. With increasing implantation energies, more and more muons reach the $\mathrm{SiC}$ bulk and with an increased fraction of $\mathrm{Mu}^{0}$ formed in the crystal, the diamagnetic signal decreases accordingly. The asymmetry data for an energy scan, taken at $10 \mathrm{~K}$ and at a magnetic field of $10 \mathrm{mT}$, is shown in Fig. 3(a).
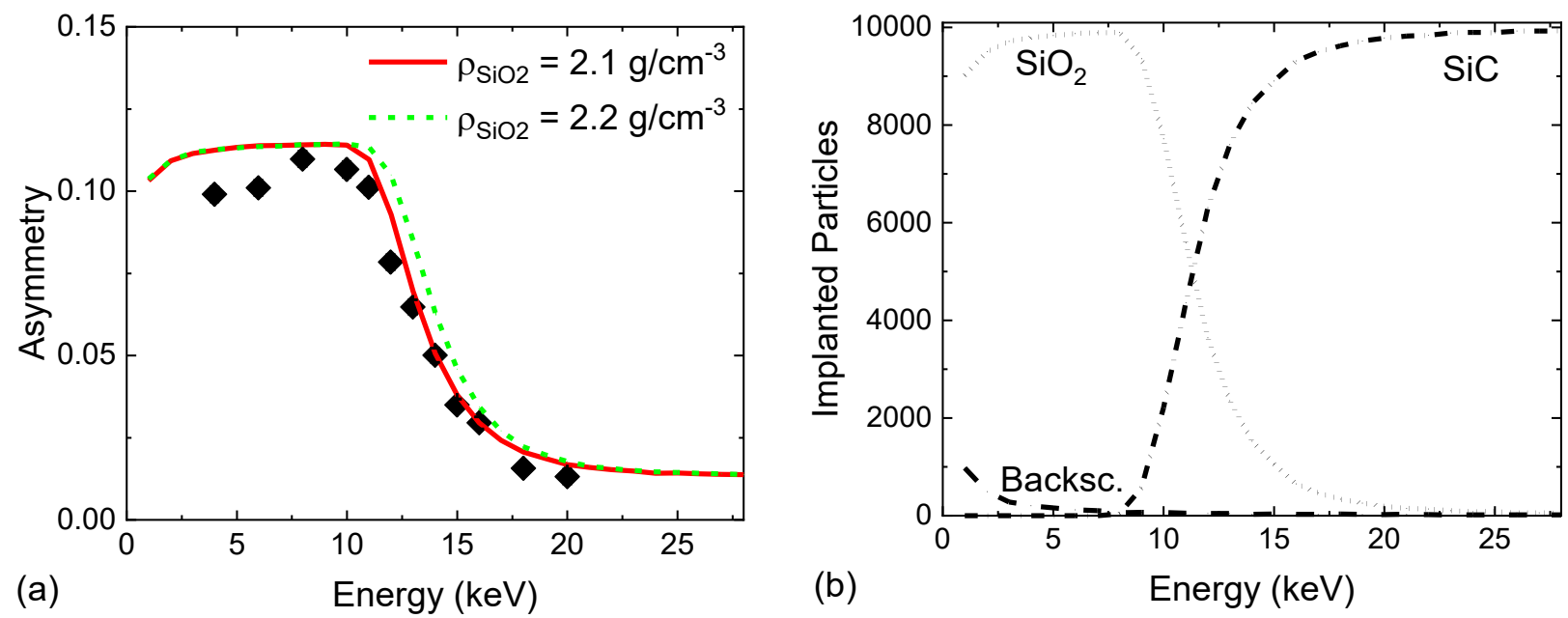

Fig. 3. (a) Diamagnetic asymmetry $A_{D}$ measured at $10 \mathrm{~K}$ and $10 \mathrm{mT}$ for the sample with a PECVDdeposited $\mathrm{SiO}_{2}$. Parametrization of the experimental data is shown as lines. Two different densities of the $\mathrm{SiO}_{2}$ layer, $\rho_{\mathrm{SiO} 2}=2.1 \mathrm{~g} / \mathrm{cm}^{-3}$ and $\rho_{\mathrm{SiO} 2}=2.2 \mathrm{~g} / \mathrm{cm}^{-3}$ are compared. (b) Muon stopping profiles which were considered for the parametrization. Depending on the implantation energy, different fractions of the muons stop either in the $\mathrm{SiO}_{2}$ or in the $\mathrm{SiC}$.

For a better understanding of the depth distribution of oxidation-induced defects, the measured asymmetries are simulated as described in Ref. [16]. For this sample with a deposited $\mathrm{SiO}_{2}$ film (Fig. 3(b)), only two layers, a low-density $\mathrm{SiO}_{2}$ layer and the $\mathrm{SiC}$ bulk are needed to describe measured $A_{D}$ values. Comparison with the observed asymmetries from the other three samples reveal very different compositions of the interface for different oxidation processes, as summarized in Table 2. In the case of $\mathrm{SiO}_{2} / 4 \mathrm{H}-\mathrm{SiC}$ interfaces with a thermally grown $\mathrm{SiO}_{2}$ (C-DRY, SiDRY+PECVD), a region with increased asymmetry is detected close to the interface, reaching both into the oxide $(\sim 15-20 \mathrm{~nm})$ and into the $\mathrm{SiC}$ bulk $(\sim 25-30 \mathrm{~nm})$. This increase of $A_{D}$ is the result of a 
suppressed $\mathrm{Mu}^{0}$ formation, which may be caused by a lack of electrons near the implanted $\mu^{+}$. Furthermore, the thermally grown oxide bulk is found to be of higher structural order than the deposited oxide, exhibiting a much higher $\mathrm{Mu}^{0}$ fraction.

Finally, the sample with a PECVD-SiO 2 layer, deposited after an etch-back of a thermally grown $\mathrm{SiO}_{2}$ layer, supports the idea of a crystal modification during thermal oxidation: no contribution of a highly defective near-interface oxide region is required to describe the measured asymmetry, while an extended layer with low $\mathrm{Mu}$ formation probability is still present in the $\mathrm{SiC}$ bulk.

The reason for the experimentally observed electron depletion or $\mathrm{SiC}$ lattice distortion due to thermal oxidation is currently still not fully understood. An emission of $\mathrm{C}$ into the SiC bulk has been proposed [18, 19]. Spectroscopic ellipsometry and capacitance-voltage studies revealed a defective or strained $\mathrm{SiC}$ layer of a few $\mathrm{nm}[19,20]$ although the extension of this defect region is an order of magnitude smaller than in our $\mu$ SR analysis. More recently, oxidation-induced local lattice distortions up to $100 \mathrm{~nm}$ were observed with x-ray diffractometry [21]. Strain could explain the observed suppression of $\mathrm{Mu}^{0}$ in the shallow $\mathrm{SiC}$ region; however, further work is needed to correlate the $\mu \mathrm{SR}$ signal to specific interface defects or other crystal deterioration mechanisms.

Table 2: Experimentally determined depth profiles of the $\mathrm{SiO}_{2} / 4 \mathrm{H}-\mathrm{SiC}$ interface for all four samples. Indicated are the extensions of interface-near regions, both in the $\mathrm{SiO}_{2}$ and in the $\mathrm{SiC}$ crystal, which exhibit an increased asymmetry compared to the bulk values.

\begin{tabular}{|l|c|l|l|}
\hline Sample Name & Oxidation Process & $\begin{array}{l}\text { SiO2, defective } \\
(\mathbf{n m})\end{array}$ & $\begin{array}{l}\text { SiC, defective } \\
(\mathbf{n m})\end{array}$ \\
\hline Si-PECVD & PECVD & $95 \mathrm{~nm}$ & - \\
\hline C-DRY & thermal & $15 \mathrm{~nm}$ & $20-25 \mathrm{~nm}$ \\
\hline Si-DRY+PECVD & thermal + PECVD & & $25-30$ \\
\hline C-DRY-PECVD & $\begin{array}{c}\text { thermal, etch back, } \\
\text { PECVD }\end{array}$ & - & $25-30 \mathrm{~nm}$ \\
\hline
\end{tabular}

\section{Summary}

In conclusion, a first proof-of-concept of LE- $\mu$ SR for the study of defects directly at or near the $\mathrm{SiO}_{2} / 4 \mathrm{H}-\mathrm{SiC}$ interface was presented. While samples with deposited $\mathrm{SiO}_{2}$ showed an abrupt transition from the oxide to the $\mathrm{SiC}$ bulk, two extended regions with lower $\mathrm{Mu}^{0}$ fractions on the oxide and the $\mathrm{SiC}$ side of the interface were observed in the case of the thermally oxidized sample. A modification of the bulk region also remained when the thermally grown oxide was etched back and a PECVD-SiO $\mathrm{S}_{2}$ layer was deposited. The observed increase in diamagnetic asymmetry at dry $\mathrm{SiO}_{2} / \mathrm{SiC}$ interfaces is believed to be closely connected to either the formation of interface defects or the introduction of lattice strain.

\section{Acknowledgements}

The muon measurements have been performed at the Swiss Muon Source S $\mu \mathrm{S}$, Paul Scherrer Institute, Villigen, Switzerland. We are grateful to Laura Maurel for performing the XRR measurements. 


\section{References}

[1] S. Dhar, S. T. Pantelides, J. R. Williams, et al., Silicon Dioxide-Silicon Carbide Interfaces: Current Status and Recent Advances, in: D. M Fleetwood and R. D. Schrimpf (Eds.), Defects in Microelectronic Materials and Devices, CRC Press, Boca Raton, 2009.

[2] M. Cabello, V. Soler, G. Rius et al., Mat. Sci. Semicon. Proc. 78, 22 (2018).

[3] B. Hornetz, H.-J. Michel, J. Halbritter, J. Mater. Res. 9, 3088 (1995).

[4] T. L. Biggerstaff, C. L. Reynolds, T. Zheleva, et al., Appl. Phys. Lett. 95, 032108 (2009).

[5] A. F. Basile, J. Rozen, J. R. Williams, et al., J. Appl. Phys. 109, 064514 (2011).

[6] X. Zhang, D. Okamoto, T. Hatakeyama, et al., Appl. Phys. Express 10, 064101 (2017).

[7] A. Yaouanc, P. D. de Rétoir, Muon spin rotation, relaxation, and resonance: applications to condensed matter, Oxford University Press, Oxford, 2011.

[8] E. Morenzoni, T. Prokscha, A. Suter, et al., J. Phys.: Condens. Matter 16, S4583 (2004).

[9] H. V. Alberto, R. C. Vilão, R. B. L. Vieira, et al., Phys. Rev. Mat. 2, 025402 (2018).

[10] B. D. Patterson, Rev. Mod. Phys. 60, 69 (1988).

[11] E. Albert, S. Barth, A. Möslang et al., Appl. Phys. Lett. 46, 759 (1985).

[12] E. Westhauser, E. Albert, M. Hamma et al., Hyperfine Interact. 32, 589 (1986).

[13] J. Woerle, T. Prokscha, A. Hallén, et al., Phys. Rev. B 100, 115202 (2019).

[14] T. Prokscha, E. Morenzoni, K. Deiters, et al., Nucl. Instrum. Meth. A 595, 317 (2008).

[15] E. Morenzoni, H. Glückler, T. Prokscha et al., Phys. B: Condens. Matter 289-290, 653 (2000).

[16] W. Eckstein, Computer Simulation of Ion-Solid Interactions, Springer Series in Material Science, Berlin, 1991.

[17] E. Morenzoni, H. Glückler, T. Prokscha et al., Nucl. Instrum. Methods Phys. Res. B 192, 254266 (2002).

[18] S. T. Dunham and J. D. Plummer, J. Appl. Phys. 59, 2551-2561 (1986).

[19] Y. Hijikata, H. Yaguchi and S. Yoshida, Mater. Sc. Forum, 679-680 (2011).

[20] H. Hashimoto, Y. Hijikata, H. Yaguchi et al., Appl. Surf. Sc. 255, 8648-8653 (2009).

[21] A. D. Hatmanto, K. Kita, Appl. Phys. Express 11, 011201 (2018). 